\title{
Interferometric characterization of the optical window for LISA Pathfinder and LISA
}

\author{
Antonio F. García Marín*, Johanna Bogenstahl*, Felipe Guzmán \\ Cervantes*, Frank Steier*, Jens Reiche*, Sascha Skorupka*, Vinzenz \\ Wand $^{*}$, Miquel Nofrarias ${ }^{\dagger}$, Josep Sanjuan ${ }^{\dagger}$, Oliver Jennrich**, Gerhard \\ Heinzel* $^{*}$ and Karsten Danzmann* \\ ${ }^{*}$ Max Planck Institute for Gravitational Physics (Albert Einstein Institute, Hanover) \\ †IEEC Barcelona, Spain \\ ${ }^{* *}$ ESTEC Noordwijk, The Netherlands
}

\begin{abstract}
In LISA Pathfinder and LISA the position fluctuations of drag free test masses will be determined interferometrically to picometer precision. To this end, laser light is brought to interference on an ultra stable optical bench after being reflected on the test mass, which needs to be in an ultra-high vacuum. The present baseline for both missions includes a separate vacuum enclosure for each test mass, so that the sensing laser beam has to pass through an optical window. This window is therefore a transmissive element in the interferometer and its associated pathlength fluctuations are potentially significant. We have selected an athermal glass that should minimize the thermally induced pathlength changes.

Several prototype windows, both mounted and unmounted, have been produced and characterized. The pathlength sensitivity to both temperature fluctuations and temperature gradients has been measured with a dedicated interferometer prototype. We have also compared the long-term stability of the LISA Technology Package interferometer when an optical window is present in the optical path to the situation without window. Finally, glass samples have been radiated and the absorption in the glass after the radiation tests has been measured to be negligible at the wavelength of interest $(1064 \mathrm{~nm})$. We present here the results of our measurements, which indicate that using a window does not influence the interferometer performance.
\end{abstract}

Keywords: Gravitational waves, interferometry, LISA, LISA pathfinder PACS: 06.30.Bp, 06.30.Gv, 07.60.Ly, 07.87.+v, 42.30.Rx, 95.55.Ym

\section{THE OPTICAL WINDOW IN THE LTP INTERFEROMETER}

In the LISA Technology Package (LTP) flying onboard LISA Pathfinder, the position fluctuations of two free-floating test masses each inside a vacuum tank are determined with a set of four interferometers and a phasemeter as described in [1].

The left part of Figure 1 shows the LTP core assembly, with each test mass inside its vacuum tank and the optical bench between them, holding the optical components that form the interferometers. The laser beam goes back and forth through the optical window (OW) into these vacuum tanks to be reflected onto the test mass. The right part of Figure 1 shows the engineering model of the optical bench with two dummy mirrors acting as test masses. This engineering model interferometer in combination with the prototype phasemeter at the Albert Einstein Institute (described in [2]) has been used for most of the investigations presented here.

CP873, Laser Interferometer Space Antenna-6 ${ }^{\text {th }}$ International LISA Symposium, edited by S. M. Merkowitz and J. C. Livas

(C) 2006 American Institute of Physics 978-0-7354-0372-7/06/\$23.00 

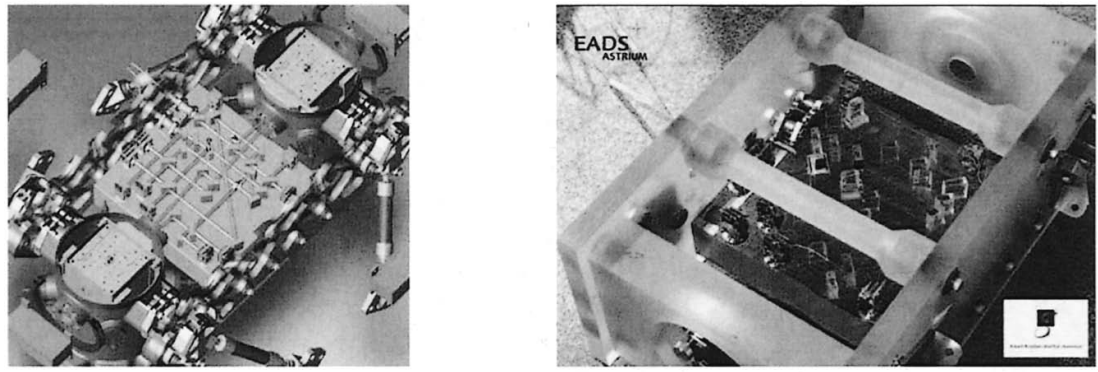

FIGURE 1. Left: CAD drawing showing a longitudinal section of the LTP core assembly. The optical bench in the center and one vacuum tank on each side enclosing the test masses. The laser beam goes back and forth through the OW into these vacuum tanks. Right: Engineering Model of the LTP optical bench with two dummy mirrors instead of test masses.

\section{THERMAL CHARACTERIZATION}

The aim of the measurements presented here is the characterization of the pathlength variations induced by environmental temperature fluctuations in the OW prototype in order to derive the required temperature stability corresponding to the allocated pathlength noise of $1 \mathrm{pm} / \sqrt{\mathrm{Hz}}$ at $1 \mathrm{mHz}$, as described in [4].


FIGURE 2. Left: Optical window prototype mounted as a transmissive element in a ULE interferometer. Right: Typical time evolution of phase and temperatures during a temperature step.

Several OW prototype have been manufactured as an athermal glass sample tightened by two titanium flanges that simulate the mechanical environment of a LTP vacuum tank. Each prototype was mounted on an dedicated ULE optical bench, similar to the LTP engineering model. Four temperature sensor and three heaters were fixed to the OW, as shown in the left part of Figure 2. Temperature steps were applied to the OW while the four temperature signals were monitored and the interferometer output phase was measured with our prototype phasemeter. The evolution of the window optical pathlength and the four temperature signals during such a step can be seen in the right part of Figure 2. 


$$
\phi=p_{1} \frac{T_{\mathrm{Ti}_{\mathrm{L}}}+T_{\mathrm{Ti}_{\mathrm{R}}}}{2}+p_{2} \frac{T_{\mathrm{Gl}_{\mathrm{L}}}+T_{\mathrm{Gl}_{\mathrm{R}}}}{2}
$$

$T_{\mathrm{Ti}_{\mathrm{L}}}:$ Temp. on left titanium sensor

$T_{\mathrm{Ti}_{\mathrm{R}}}$ : Temp. on right titanium sensor

$T_{\mathrm{Gl}_{\mathrm{L}}}$ : Temp. on left glass sensor

$T_{\mathrm{Gl}}$ : Temp. on right glass sensor

\begin{tabular}{|c|c|}
\hline & BB1 \\
\hline $\mathrm{p}_{1}[\mathrm{rad} / \mathrm{K}]$ & $0.09 \pm 0.01$ \\
\hline $\mathrm{p}_{2}[\mathrm{rad} / \mathrm{K}]$ & $-0.061 \pm 0.06$ \\
\hline
\end{tabular}

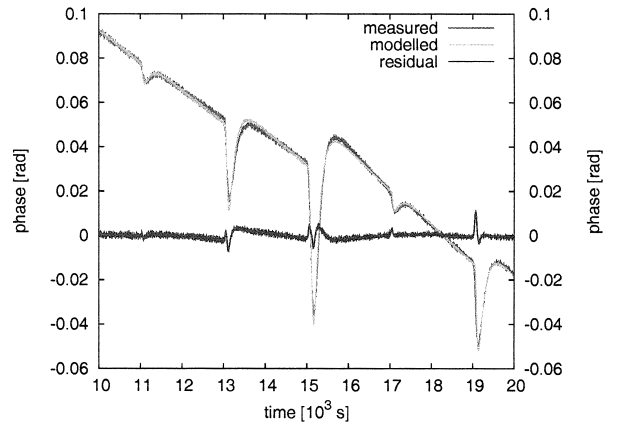

FIGURE 3. Left: model of the optical pathlength of the window $(\varphi)$ as function of the temperature in the glass and in the titanium. Right: Time evolution of the interferometric phase during several temperature pulses showing the agreement between the measured optical pathlength and the modelled one.

The interferometric phase was then fitted to the model shown in the left part of Figure 3 (after removing a quadratic drift). Thermal induced pathlength variations have been modelled to account for $\approx 100 \mathrm{mrad} / \mathrm{K}$ and no significantly larger effect from thermal gradients have been observed. Taking as an example a budget of $1 \mathrm{pm} / \sqrt{\mathrm{Hz}}$ (approx. $6 \mu \mathrm{rad} / \sqrt{\mathrm{Hz}}$ ) would lead to a required environmental thermal stability of $1.2 \times$ $10^{-5} \mathrm{~K} / \sqrt{\mathrm{Hz}}$ in the measurement band.

\section{LONG-TERM INTERFEROMETRIC PERFORMANCE}

The long-term behavior of the OW was tested with the engineering model of the LTP interferometer shown in Figure 1. The aim of the measurements is to investigate an eventual degradation of the interferometric performance when the $\mathrm{OW}$ is present in the beam path.

To this end, the OW prototype was attached to the side of the optical bench plate in front of the dummy mirror that acts as test mass 2 as shown in the left part of Figure 4. The readout of test mass 1 position fluctuations is an independent indicator of the interferometric performance. Three of the tested OW prototypes had an anti-reflex coating applied on both sides of the athermal glass (to investigate mechanical stress induced by the coating) and had undergone various environmental tests (leak testing, random vibration and thermal cycling).

Several performance measurements have been done on each OW sample. A typical result (right part of Figure 4) shows the performance of the X12 interferometer (where an OW prototype has been placed in front of the dummy mirror acting as test mass 2) compared to the performance of the X1 interferometer (without OW). The third curve shows the best sensitivity measured with this system (engineering model of the optical bench and our prototype phasemeter, before any OWs were introduced). No degradation of the interferometric readout was detected, thus settling effects from the environmental 

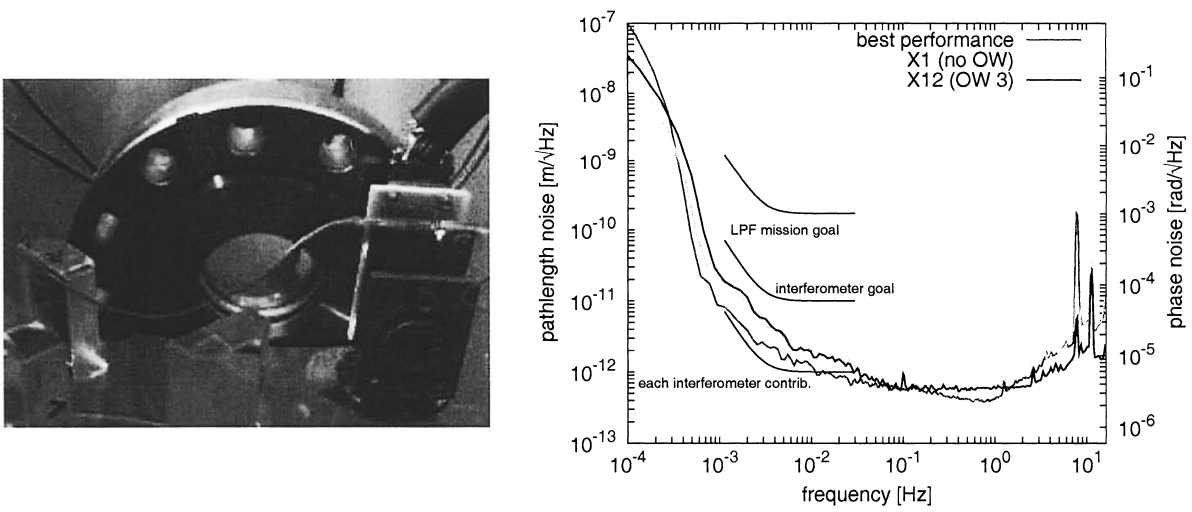

FIGURE 4. Left: OW prototype attached to the engineering model of the optical bench (shown in Figure 1) in front of the dummy mirror that acts as test mass 2 . Right: Sensitivity to position fluctuations of the test masses measured with the engineering model interferometer at the Albert Einstein Institute


FIGURE 5. Left: Schematic view of one glass sample to be radiated in four different spots with different dose. Right: Sample showing the strongest darkening in the spot radiated with $200 \mathrm{krad}$.

testing or stress effects induced by anti-reflex coating could be discarded.

\section{RADIATION TESTS}

We present here investigations about the potential degradation of the glass optical properties due to the environmental radiation during the LTP mission time. The absorbed dose has been estimated in [5] to be $\mathbf{1 2} \mathbf{~ k r a d}$.

Four glass samples were exposed to an equivalent absorbed dose between $1 \mathrm{krad}$ and $200 \mathrm{krad}$ using a $30 \mathrm{MeV}$ proton beam as described in [4]. Each sample was irradiated in four different locations, as shown in the left part of Figure 5. The darkening observed in the right part of Figure 5 is due to the appearance of color-centers, defects caused by the interaction of high energy particles with the material structure.

In order to evaluate quantitatively the absorption caused by the color centers in the 

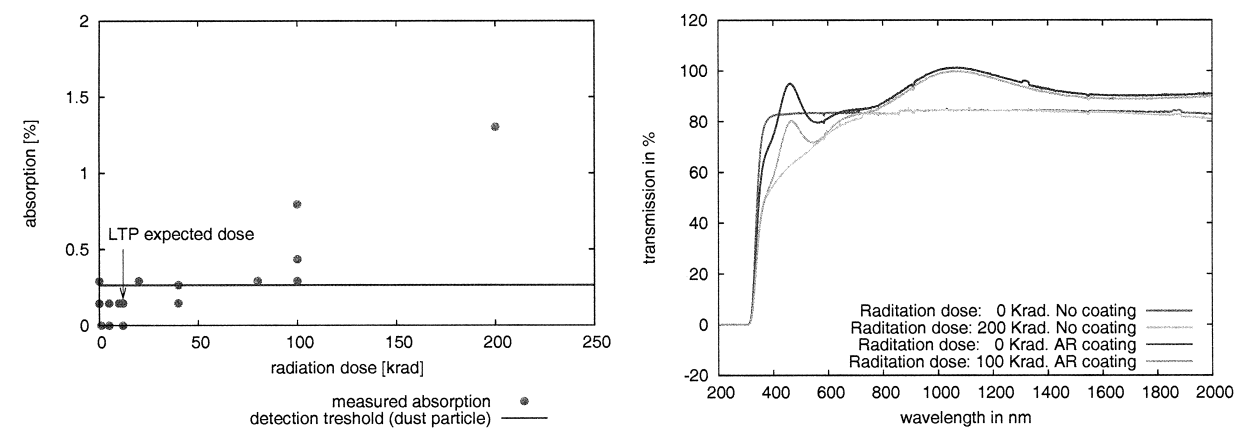

FIGURE 6. Left: Relative absorption at $1064 \mathrm{~nm}$ for different radiation dose. Right: Transmission measured in a wide spectral range for two samples with and without anti-reflex coating.

radiated samples, two different type of measurements of the light transmission through the samples were performed.

The left part of Figure 6 shows the transmission as function of the radiation dose measured with a Nd-YAG laser with the same properties (wavelength, polarization and intensity) as in LTP. The measured absorption remains under $1.4 \%$.

Measurements of the relative transmission were also performed as function of the wavelength with a spectrometer (Perkin Elmer UV/VIS/NIR Spectrometer Lambda 900). The right part of Figure 6 shows the spectra of an AR coated and an uncoated sample in two different spots: the darkest spot $(200 \mathrm{krad})$ and a non-radiated area. The two spectra of the AR-coated window can be recognized due to its non-flat, improved transmissivity around $1064 \mathrm{~nm}$. In both samples, the absorption due to the radiation is only measurable for wavelengths well below $1064 \mathrm{~nm}$, from $800 \mathrm{~nm}$ down to UV. This explains the apparent brown color of the samples despite the measured negligible absorption at $1064 \mathrm{~nm}$.

\section{ACKNOWLEDGMENTS}

We would like to acknowledge the Hahn-Meitner-Institut in Berlin, where the proton irradiation tests were done. Also Carlo Gavacci Space for assembling the OW prototypes.

\section{REFERENCES}

1. G. Heinzel et al 2003 Class. Quantum Grav. $20153-161$

2. G. Heinzel et al 2004 Class. Quantum Grav. 21 581-587

3. G. Heinzel, "S2-AEI-TN-3016 Requirements on LPF optical windows." LTP project technical note.

4. M. Nofrarias, A. F. García Marín, J. Sanjuan, "S2-IEEC-TN-3019 Thermal test on optical window." LTP project technical note.

5. H. G. Grothues "S2-DLR-TN-3001 Preparation of Particle Irradiation Tests of OHARA S-PHM52 Optical Glass Windows." LTP project technical note. 